\section{References}

1. McGoon DC, Rastelli GC, Ongley PA. An operation for the correction of truncus arteriosus. JAMA. 1968;205:69-73.

2. Sinzobahamvya N, Asfour B, Boscheinen M, Photiadis J, Fink C, Schindler E, et al. Compared fate of small-daimeter Contegras and homografts in the pulmonary position. Eur J Cardiothorac Surg. 2007;32:209-14.
3. Lecompte Y, Neveux JY, Leca F, Zannini L, Tu TV, Duboys Y, et al. Reconstruction of the pulmonary outflow tract without prosthetic conduit. J Thorac Cardiovasc Surg. 1982;84:727-33.

4. Nakae S, Kawada M, Kasahara S, Kuroyama N, Hiraishi S, Yoshimura H. Correction of truncus arteriosus with autologous arterial flap in neonates and small infants. Ann Thorac Surg. 1996;62:123-9.

5. Haydar S. Lecompte maneuver in truncus arteriosus repair: potential risks! Ann Thorac Surg. 1996;62:1241.

\title{
Pediatric Application of the Thoratec CentriMag BiVAD as a Bridge to Heart Transplantation
}

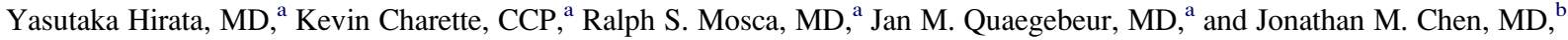 \\ New York, NY
}

The Thoratec CentriMag ventricular assist device (VAD) (Thoratec Corporation Pleasanton, Calif) (Figure 1) is a centrifugal pump designed for extracorporeal support that operates without mechanical bearings or seals. Although extracorporeal membrane oxygenation (ECMO) provides cardiac and respiratory support in a rapid manner for the pediatric patient population, it offers only short-term support with an increase in complications beyond the second week. The CentriMag system has advantages over other systems: the pumps have a low priming volume and low requirements for heparin and hemolysis. We report three successful applications of the CentriMag BiVAD as a bridge to pediatric heart transplantation.

\section{CLINICAL SUMMARIES}

PATIENT 1. A 7-year-old previously healthy boy with a 10day history of abdominal symptoms was brought to the emergency department initially stable, but his condition deteriorated and later necessitated intubation and full cardiac support; his oxygen saturation decreased to $40 \%$. Echocardiographic analysis revealed severely decreased left ventricular function. The heart arrested in the operating room before incision and cardiopulmonary bypass was instituted as an emergency measure. A malleable $28 \mathrm{~F}$ lighthouse tip

From the Division of Cardiothoracic Surgery, Department of Surgery, Columbia University College of Physicians and Surgeons, ${ }^{a}$ and the Department of Cardiothoracic Surgery, Joan and Sanford Weill Medical College of Cornell University, ${ }^{\text {b }}$ New York, NY.

Received for publication Nov 1, 2007; revisions received Feb 29, 2008; accepted for publication May 4, 2008.

Address for reprints: Yasutaka Hirata, MD, Pediatric Cardiac Surgery, Columbia University College of Physicians and Surgeons, New York, NY (E-mail: yh2240@ columbia.edu).

J Thorac Cardiovasc Surg 2008;136:1386-7

$0022-5223 / \$ 34.00$

Copyright (c) 2008 by The American Association for Thoracic Surgery doi:10.1016/j.jtcvs.2008.05.028

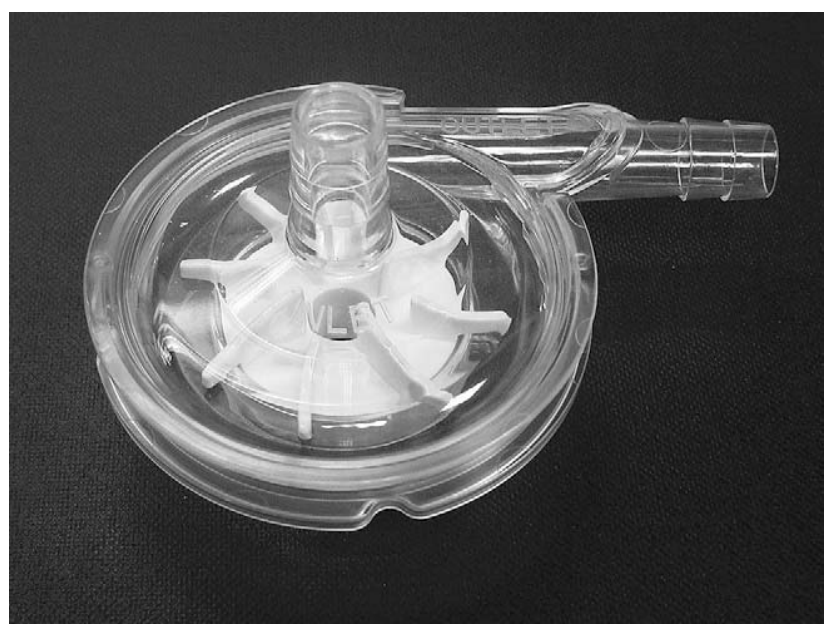

FIGURE 1. CentriMag pump.

cannula (Medtronic, Inc, Minneapolis, Minn) was placed through an incision along the costal margin at a point just inferior to the approximate site of the left ventricular apex. Two concentric purse-string sutures of 3-0 Prolene polypropylene (Ethicon, Inc, Somerville, NJ) supported with bovine pericardial pledgets were placed around the left ventricular apex, and the inflow cannula was placed into the left ventricle. Two 14F Biomedicus cannulas (Biomedicus, Inc, Eden Prairie, Minn) were similarly placed through two incisions along the epigastrium and inserted into the ascending aorta and main pulmonary artery. A 24F malleable lighthouse tip cannula was placed through an incision along the right thoracic wall, and the inflow cannula was placed into the right atrium, directed toward the inferior vena cava. Heparin was started at 24 hours to maintain an activated clotting time of 160 to 180 seconds. Because of pulmonary edema and desaturation, an oxygenator was inserted in-line with the right VAD outflow and was removed after 24 hours once his 
TABLE 1. Clinical summary of the 3 patients

\begin{tabular}{llll}
\hline & \multicolumn{1}{c}{ Patient 1 } & \multicolumn{1}{c}{ Patient 2 } & Patient 3 \\
\hline Body weight $(\mathrm{kg})$ & 32 & 39.8 & 10.2 \\
LVAD flow (mL/min) & $1.9-2.1$ & $3.0-3.1$ & $0.9-1.0$ \\
RVAD flow (mL/min) & $1.9-2.1$ & $3.0-3.1$ & $0.8-1.0$ \\
Cannulas & & & $22 \mathrm{~F}$ malleable lighthouse tip \\
LVAD inflow & 28F malleable lighthouse tip & 28F malleable lighthouse tip & $12 \mathrm{~F}$ Biomedicus \\
LVAD outflow & 14F Biomedicus & 14F Biomedicus & $22 \mathrm{~F}$ malleable lighthouse tip \\
RVAD inflow & 24F malleable lighthouse tip & 28F malleable lighthouse tip & $12 \mathrm{~F}$ Biomedicus \\
RVAD outflow & 14F Biomedicus & $14 \mathrm{~F}$ Biomedicus & $8 \mathrm{~h}$ \\
Transplantation & $4 \mathrm{~d}$ & $3 \mathrm{~d}$ & Alive \\
Outcome & Alive & Alive &
\end{tabular}

oxygenation had improved with diuresis. He underwent successful heart transplantation 4 days later.

PATient 2. A 10-year-old boy with history of dilated cardiomyopathy underwent CentriMag BiVAD implantation and successful heart transplantation 3 days later.

PATient 3. A 3-year-old boy with a history of hereditary cardiomyopathy underwent CentriMag BiVAD implantation. At 24 hours postoperatively he was noted to have a small amount of thrombus in the inflow tubing to the device and thus had his left-sided pump exchanged at the bedside. He underwent successful transplantation 8 hours later.

Table 1 shows the clinical summary of the patients.

\section{DISCUSSION}

Although ECMO is still the most common form of mechanical support used for infants and young children, data from several institutions suggest ECMO is effective in bridging only half of the eligible children to heart transplantation. ${ }^{1-3}$ Complications such as multiorgan failure, sepsis, and thromboembolism continue to limit ECMO survival to 3 or 4 weeks. The CentriMag BiVAD is technically easy to implant, uses any commercially available inflow or outflow cannulas, and flows between $200 \mathrm{~mL} / \mathrm{min}$ and $8 \mathrm{~L} /$ min. The pump also allows both the potential for extubation and the possibility of including an oxygenator in-line with the right-sided device if necessary, as in our first case.

We have noted several salient points when implanting the CentriMag VAD in children as opposed to adults. First, because of the often very lateral location of the left ventricular apex and the acute angle of the costal margin in children, it is most useful to insert the inflow cannulas through the intercostal spaces with a short subcutaneous tunnel.
Second, because the hearts of these children seem to dilate disproportionately to the size of the great vessels, often the outflow cannulas must traverse a broad angle (and long distance) over the shoulder of the right ventricle before being inserted into the great vessels (which are small) at a nearly $90^{\circ}$ angle. It is therefore important not to insert the cannula too far, inasmuch as it will abut the back wall of the great vessel and cause outflow resistance.

Finally, because of the lower flows required with smaller children, an aggressive anticoagulation scheme is suggested, with the commencement of heparin therapy soon after the implantation. Additionally, the use of heparin-coated connectors and tapered tubing, while untested, seems intuitively logical. We also run below full flow in smaller patients so as to decrease the likelihood of obliteration of the left ventricular cavity and air entrainment.

\section{CONCLUSION}

We report the successful use of the CentriMag BiVAD in 3 children bridged to transplantation. This clinical indication awaits further application in this age group to better define the possibility of longer term support.

\section{References}

1. Fiser WP, Yetman AT, Gunselman RJ, Fasules JW, Baker LL, Chipman CW, et al Pediatric arteriovenous extracorporeal membrane oxygenation (ECMO) as a bridge to cardiac transplantation. J Heart Lung Transplant. 2004;22:770-7.

2. Gajarski RJ, Mosca RS, Ohye RG, Bove EL, Crowley DC, Custer JR, et al. Use of extracorporeal life support as a bridge to pediatric cardiac transplantation. J Heart Lung Transplant. 2004;22:28-34.

3. Kirshbom PM, Bridges ND, Myung RJ, Gaynor JW, Clark BJ, Spray TL. Use of extracorporeal membrane oxygenation in pediatric thoracic organ transplantation. J Thorac Cardiovasc Surg. 2002;123:130-6. 